\title{
Bilateral Hippocampal Infarction as Etiology of Sudden and Prolonged Memory Loss
}

\author{
Ivan Marinkovic $^{a} \quad$ Jukka Lyytinen $^{\mathrm{a}}$ Leena Valanne $^{\mathrm{b}}$ \\ Riikka Niinikuru $^{a}$ Eero Pekkonen ${ }^{a}$ \\ Departments of ${ }^{\mathrm{a}}$ Neurology and ${ }^{\mathrm{b}}$ Radiology, Helsinki University \\ Central Hospital, Helsinki, Finland
}

\section{Key Words}

Transitory global amnesia - Hippocampal infarction - Retro- and anterograde amnesia · Ischemic stroke

\begin{abstract}
Sudden memory loss, with prolonged cognitive deterioration, clinically initially resembling a transitory global amnesia (TGA)-like episode, might be caused by ischemic stroke in the hippocampal regions. We report a patient with TGA-type sudden anterograde amnesia and normal head CT. Examinations revealed that the patient had several vascular risk factors and 3 tesla (T) head MRI showed ischemic lesions in diffusion-weighted images (DWI) in both hippocampi. Neuropsychological assessment revealed sustained moderate verbal memory deterioration and abnormal executive functions. We suggest that small ischemic strokes in hippocampal regions might remain unrecognized and underdiagnosed if follow-up of TGAtype episodes is not adequate and if head CT remains the only method of brain imaging.
\end{abstract}

\section{Introduction}

The vascular supply of the hippocampus originates from several hippocampal arteries coming from the posterior cerebral artery, although its anterior part is mainly supplied by the anterior choroidal artery which typically originates from the posterior wall of the internal carotid artery [1]. Uni- and bilateral hippocampal infarctions are directly involved in memory dysfunction [2,3], with potential to cause irreversible amnesia. 
Transient global amnesia (TGA) has been described as a reversible inability to store or retrieve information, lasting less than $24 \mathrm{~h}$. Most TGA attacks, however, typically subside in 4-6 $\mathrm{h}$ [4]. An ischemic nature of TGA involving both hippocampi, based on diffusion-weighted images (DWI) and MRI data, has recently been proposed [5].

\section{Patient and Methods}

Our patient is a 66-year-old woman, non-smoker with a history of hypertension, and neither other diseases nor regular medication.

After performing regular morning activities, the patient had a sudden onset of anterograde memory loss. Upon arrival at the emergency department of Helsinki University Central Hospital, she also complained of headache and numbness in the fingers. No other neurological deficits were noticed. The patient had neither fever nor other signs of infection.

Head CT, CT-angiography of intracranial arteries, electroencephalography (EEG) as well as chest Xray and cardiac ultrasound were all normal. All laboratory tests (including a CRP value of $<3 \mathrm{mg} / \mathrm{l}$ and a blood leukocyte count of $8.4 \times 10^{9} / 1$ ) were unremarkable. Because symptoms remained beyond $24 \mathrm{~h}$, a 3-T head MRI was done, revealing small dot-like ischemic lesions in DWI sequences in both hippocampi (fig. 1 fig. 2 fig. 3 ). A Doppler ultrasound found plaques in vertebral arteries. Total cholesterol was $5.5 \mathrm{mmol} / \mathrm{l}$, and LDL was $2.4 \mathrm{mmol} / \mathrm{l}$. High/raised blood pressure levels were observed (>140/90 mm Hg). Antihypertensive medication and cholesterol and antiplatelet agents were started.

\section{Results}

An orienting neuropsychological assessment was performed 10 days after hospitalization. A memory impairment varying from slight to moderate in nature was the major finding. A moderate verbal memory deficit was observed. Immediate recall (story recall, list learning) was moderately to severely impaired and subject to intrusions. Regarding delayed verbal memory, spontaneous retrieval was defective but recognition memory was intact. Furthermore, minor impairments were observed in verbal working memory. As for visual memory, mild impairments in both immediate and delayed recall were detected. In addition, apparent difficulties in naming were found. Regarding executive functions, difficulties in planning and monitoring, and a perseveration tendency were found. A minor impairment in attention, namely inattentiveness was observed. Symptom awareness was slightly exaggerated.

\section{Discussion}

TGA was the primary diagnosis, based on the patient's initial condition and supported by normal head CT. However, the patient's cognition remained impaired for more than $24 \mathrm{~h}$, which clinically did not fit into the generally accepted duration of TGA symptoms [6].

Our patient had detectable deficits in neuropsychological testing performed 10 days after symptoms onset, whereas in TGA, all, or most, cognitive changes are expected to vanish within $24 \mathrm{~h}$ [7]. According to some reports, mild impairment in verbal memory has been observed after long-term follow-up of TGA patients [2]. In contrast, our patient's neuropsychological assessment also demonstrated other cognitive deficits and 
mood changes that persisted for a considerable time period (more than 10 days after initial symptom onset).

In a meta-analysis comprising 374 TGA patients [8], it was concluded that during the first $24 \mathrm{~h}$ after symptom onset there were impairments especially in anterograde and retrograde episodic long-term memory as well as executive functions. Similar, but smaller differences were found for as long as 5 days after onset of an episode.

Thereafter (5-30 days after a TGA episode), no significant differences between patients and controls were found in any cognitive domains.

Our patient underwent brain MRI ( $48 \mathrm{~h}$ after symptom onset) because the cognitive symptoms did not resolve. Our findings are in concordance with a previous report [9]. Further, patients with a clinical diagnosis of TGA have normal brain MRI findings [1013]. Cases with persistent memory impairment after hippocampal region strokes have also been described [14]. The ischemic etiology was supported by the patient's vascular risk factors.

MRI lesions did not have features generally observed after prolonged epileptic activity, such as diffuse cortical hyperintensity in DWI sequences, which would correlate to nonconvulsive epileptic status [15]. Vasogenic edema, which would refer to venous thrombosis, was not present, and occlusion of neither cerebral arteries nor the venous system was observed in MR angiography images. Sudden onset of symptoms, with neither fever nor other signs of infection, normal EEG, and MRI lesions typical for ischemia made encephalitis clinically highly unlikely. Therefore, lumbar puncture was not performed.

We suggest that in patients with normal head CT and unresolved anterograde amnesia resembling TGA, especially those carrying significant vascular risk factors, a head MRI scan should be done in order to reveal possible hippocampal infarctions.

\section{Acknowledgements}

We thank Dr Filip Scheperjans for excellent technical assistance in image processing.

\section{Disclosure Statement}

The authors have no conflict of interest to disclose. 

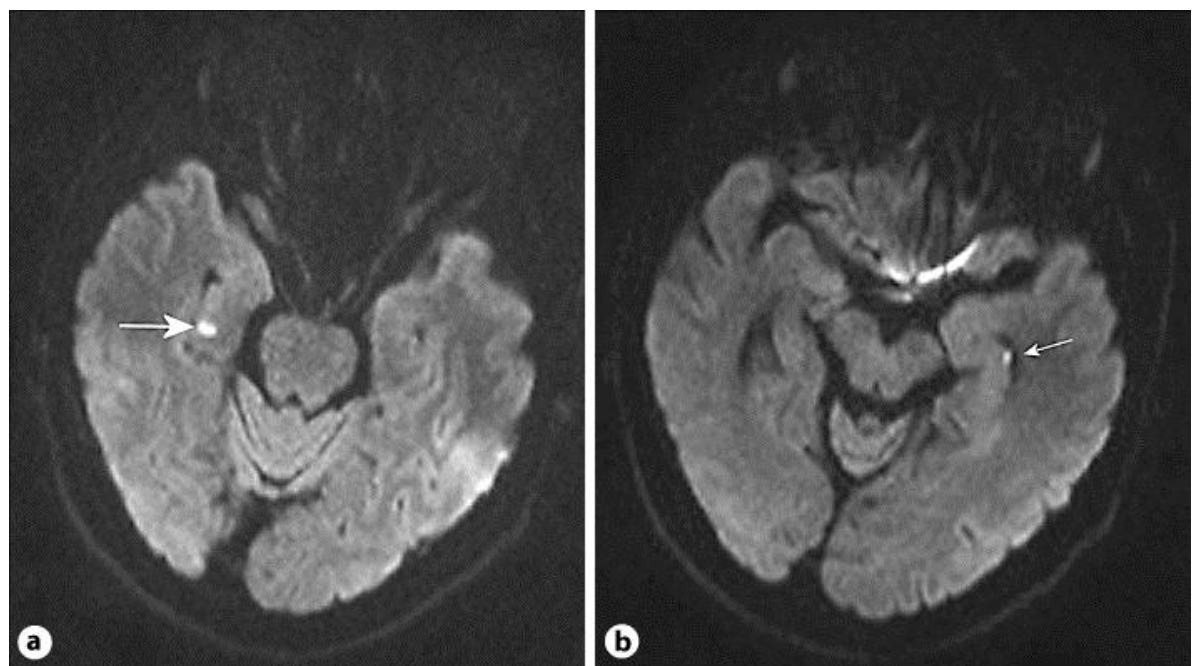

Fig. 1. a, b DWI shows small infarctions in both hippocampi (arrows). Small lesions were seen in both hippocampi, hyperintense in T2-weighted images and showing restricted diffusion in DWI. The rest of the hippocampi were normal, without any signs of encephalitis.

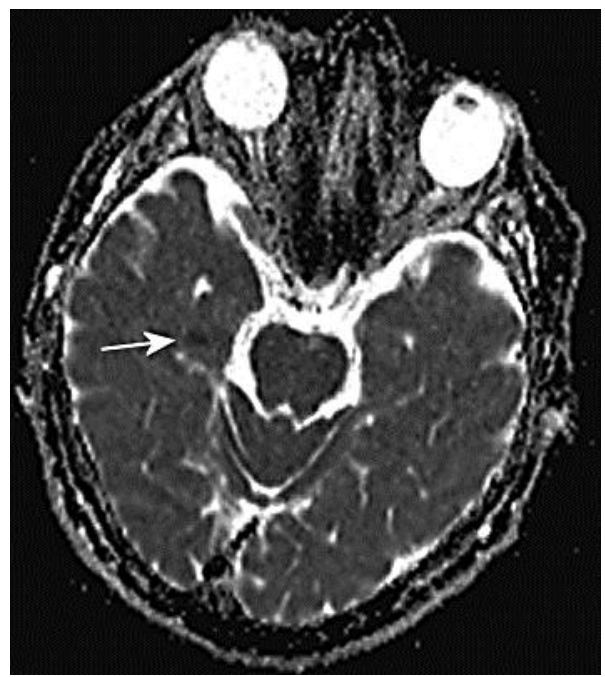

Fig. 2. ADC map confirms the ischemic finding on the right to be due to restricted diffusion (arrow). A similar finding was also seen on the left (not shown). 


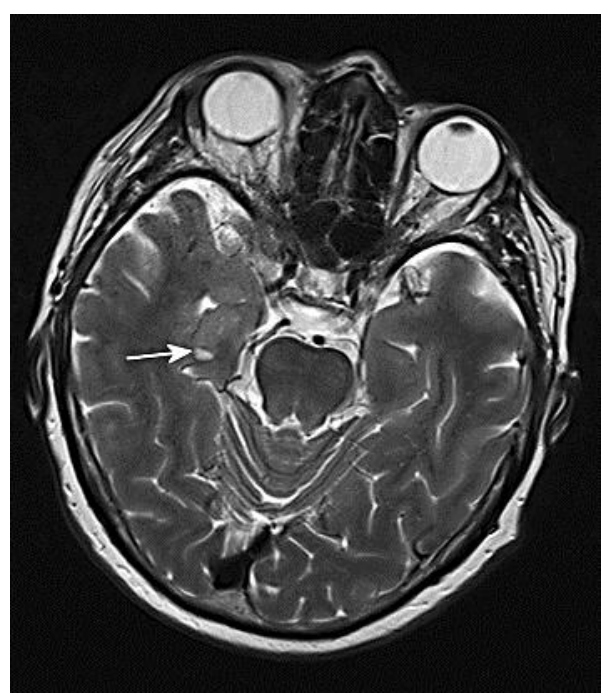

Fig. 3. T2-weighted image shows a small lesion (arrow) corresponding to the DWI findings but does not reveal any other abnormalities in the right hippocampus.

\section{References}

1 Muller J, Shaw L: Arterial vascularization of the human hippocampus. 1. Extracerebral relationships. Arch Neurol 1965;13:45-47.

2 Borroni B, et al: Is transient global amnesia a risk factor for amnestic mild cognitive impairment? J Neurol 2004;251:1125-1127.

-3 Takahashi S, et al: Correlation of lesions in the hippocampal region noted on MR images with clinical features. Eur Radiol 1997;7:281-286.

4 Zeman AZ, Hodges JR: Transient global amnesia. Br J Hosp Med 1997;58:257-260.

5 Liang JF, Shen AL, Lin SK: Bilateral hippocampal abnormalities on diffusion-weighted MRI in transient global amnesia: report of a case. Acta Neurol Taiwan 2009;18:127-129.

-6 Hodges JR, Warlow CP: The aetiology of transient global amnesia. A case-control study of 114 cases with prospective follow-up. Brain 1990;113(Pt 3):639-657.

-7 Regard M, Landis T: Transient global amnesia: neuropsychological dysfunction during attack and recovery in two 'pure' cases. J Neurol Neurosurg Psychiatry 1984;47:668-672.

8 Jager $\mathrm{T}$, et al: The transience and nature of cognitive impairments in transient global amnesia: a metaanalysis. J Clin Exp Neuropsychol 2009;31:8-19.

-9 Sedlaczek 0, et al: Detection of delayed focal MR changes in the lateral hippocampus in transient global amnesia. Neurology 2004;62:2165-2170.

10 Förster A, et al: Diffusion-weighted imaging for the differential diagnosis of disorders affecting the hippocampus. Cerebrovasc Dis 2012;33:104-115.

11 Budson AE, Schlaug G, Briemberg HR: Perfusion- and diffusion-weighted magnetic resonance imaging in transient global amnesia. Neurology 1999;53:239-240.

12 Gass A, et al: Lack of evidence of acute ischemic tissue change in transient global amnesia on single-shot echo-planar diffusion-weighted MRI. Stroke 1999;30:2070-2072.

13 Huber R, et al: Transient global amnesia. Evidence against vascular ischemic etiology from diffusion weighted imaging. J Neurol 2002;249:1520-1524.

14 Perez-Lazaro C, et al: Amnesic stroke caused by hippocampal infarction (in Spanish). Rev Neurol 2005;41:27-30.

15 Chu K, et al: Diffusion-weighted magnetic resonance imaging in nonconvulsive status epilepticus. Arch Neurol 2001;58:993-998. 